ELECTRONIC RESEARCH ANNOUNCEMENTS OF THE AMERICAN MATHEMATICAL SOCIETY

Volume 4, Pages 4-11 (March 18, 1998)

S $1079-6762(98) 00040-7$

\title{
PRESCRIBING MEAN CURVATURE: EXISTENCE AND UNIQUENESS PROBLEMS
}

\author{
G. KAMBEROV
}

\begin{abstract}
The paper presents results on the extent to which mean curvature data can be used to determine a surface in $\mathbf{R}^{\mathbf{3}}$ or its shape. The emphasis is on Bonnet's problem: classify and study the surface immersions in $\mathbf{R}^{\mathbf{3}}$ whose shape is not uniquely determined by the first fundamental form and the mean curvature function. These immersions are called Bonnet immersions. A local solution of Bonnet's problem for umbilic-free immersions follows from papers by Bonnet, Cartan, and Chern. The properties of immersions with umbilics and global rigidity results for closed surfaces are presented in the first part of this paper.

The second part of the paper outlines an existence theory for conformal immersions based on Dirac spinors along with its immediate applications to Bonnet's problem. The presented existence paradigm provides insight into the topology of the moduli space of Bonnet immersions of a closed surface, and reveals a parallel between Bonnet's problem and Pauli's exclusion principle.
\end{abstract}

\section{INTRODUCTION}

The results in this paper are part of a program to understand to what extent one can use mean curvature data to determine the shape of a surface in $\mathbf{R}^{3}$.

The first section of the paper presents results on a problem suggested by Bonnet. A generic immersion is uniquely determined up to a rigid motion by its first fundamental form and its mean curvature function, but there are some exceptions, for example most constant mean curvature immersions. Bonnet's problem is to classify and study all exceptional immersions. Here I concentrate on the study of Bonnet's problem for immersions with umbilic points and immersions of closed surfaces.

The second section contains an outline of an existence theory for conformal immersions ([KNPP, Pe, Pi1, Ka1]) along with its immediate applications to Bonnet's problem. The central idea in the theory is to define square roots of basic geometric objects, for example, area elements, differentials of maps, and then determine the equations satisfied by the square root of the differential of a conformal immersion. From an analytic point of view the main advantage of the approach is that the existence problem for prescribing mean curvature data is reduced to a first order elliptic system. The theory suggests a new paradigm: the data used to determine the immersion are the conformal structure, the regular homotopy class of the immersion, and the mean curvature half-density, that is, the mean curvature

Received by the editors February 27, 1996 and, in revised form, January 1, 1998.

1991 Mathematics Subject Classification. Primary 53C42; Secondary 35Q40, 53A05, 53A30, 53A50, 58D10, 81Q05.

Key words and phrases. Surfaces, spinors, conformal immersions, prescribing mean curvature.

(C)1998 American Mathematical Society 
appropriately weighted by the metric. These data determine the immersion via a generalized Weierstrass-Kenmotsu formula.

Robert Bryant introduced me to Bonnet's problem. I thank him and Ulrich Pinkall, Franz Pedit, Peter Norman, and Dennis DeTurck for many discussions, advice, and support.

\section{BONNET'S UNIQUENESS PROBLEM}

Two isometric immersions $F_{1}$ and $F_{2}$, of a given Riemann surface $(M, g)$ into $\mathbf{R}^{3}$, are called congruent (denoted by $F_{1} \sim F_{2}$ ) if they differ by a rigid motion. I am interested in the following questions: What conditions guarantee that $H_{1}=$ $H_{2}$ implies $F_{1} \sim F_{2}$ ? What can be said if $H_{1}=H_{2}$, but $F_{1}$ and $F_{2}$ are not congruent? The isometric immersions $F_{1}$ and $F_{2}$ are called Bonnet mates if they are not congruent, but their mean curvature functions, $H_{1}$ and $H_{2}$, are equal.

Bonnet, Cartan, and Chern studied the existence and classification of Bonnet mates. Their works yield a complete local classification of the umbilic-free immersions which admit Bonnet mates ([Bon, Car, Che]). Some global results were obtained by Lawson and Tribuzy, and by Ros (see [LT] and [Ros]).

The methods used in [Bon, Car], and [Che] are very powerful and beautiful but cannot be used to study nonconstant mean curvature surfaces with umbilic points. Until recently it was unknown whether such Bonnet mates exist. In particular, it was unknown whether there are any Bonnet mates which are not included in the Bonnet-Cartan-Chern classification. A general construction which yields all immersions, with or without umbilic points, that admit Bonnet mates was found in $[\mathrm{KPP}]$. This construction made it possible to prove that there exist infinitely many new Bonnet immersions, that is, Bonnet mates which have umbilic points and whose mean curvature is not constant in a neighborhood of the umbilic points ([Ka1] and [Ka2]).

This section contains results on the properties of the umbilic points of Bonnet mates and on the the following rigidity conjecture: Let $(M, g)$ be a closed oriented Riemann surface and let $H: M \rightarrow \mathbf{R}$ be an arbitrary function. Then up to rigid motions, there exists at most one isometric immersion of $(M, g)$ into $\mathbf{R}^{\mathbf{3}}$ with mean curvature function $H$.

Throughout this note $(M, g)$ is an oriented, connected Riemann surface; $F_{1}$, $F_{2}$ are isometric immersions of $(M, g)$ in $\mathbf{R}^{\mathbf{3}} ; I I_{i}, N_{i}$, and $H_{i}$ denote the second fundamental form, the field of unit normals, and the mean curvature function of the immersion $F_{i}$. The mean curvatures of two isometric immersions $F_{1}$ and $F_{2}$ coincide if and only if $F_{1}$ and $F_{2}$ have the same principal curvatures at every point, and so if $H_{1}=H_{2}$, then $p$ is an umbilic for one of the immersions precisely if it is an umbilic for the other.

It is convenient to introduce the shape distortion tensor, $D=I I_{1}-I I_{2}$, associated with $F_{1}$ and $F_{2}$. Thus, Bonnet's fundamental theorem of surface theory implies that $F_{1} \sim F_{2}$ if and only if $D \equiv 0$. The Mainardi-Codazzi equations imply the following observation:

Observation 1.1. If the mean curvature functions of $F_{1}$ and $F_{2}$ coincide, then the associated shape distortion operator is trace-free and divergence-free, i.e, its $(2,0)$ part, $D^{2,0}$, is holomorphic.

Observation 1.1 implies the following unique continuation property: 
Corollary 1.1. Let $F_{1}: M \rightarrow \mathbf{R}^{\mathbf{3}}$ and $F_{2}: M \rightarrow \mathbf{R}^{\mathbf{3}}$ be isometric embeddings which have the same principal curvatures. If $F_{1} \sim F_{2}$ on a nonempty open set, then $F_{1} \sim F_{2}$ on $M$.

Analyzing the shape distortion tensor yields the following theorem describing the properties of the umbilic points of a Bonnet immersion.

Theorem 1. Suppose that $F_{1}$ and $F_{2}$ are Bonnet mates; then

A. $D_{p}=0$ if and only if $p$ is umbilic; moreover, for every umbilic point $p$ we have

$$
\operatorname{ind}_{F_{1}}(p)=\operatorname{ind}_{D^{2,0}}(p)=\operatorname{ind}_{F_{2}}(p)<0,
$$

where $\operatorname{ind}_{F_{1}}(p)$ and $\operatorname{ind}_{F_{2}}(p)$ denote the index of $p$ with respect to $F_{1}$ and $F_{2}$, respectively, and $\operatorname{ind}_{D^{2,0}}(p)$ is the index of $p$ with respect to the horizontal foliation of the quadratic differential $D^{2,0}$. (See [Hopf] for the definitions of the different notions of index.)

B. Every umbilic point is a critical point of the mean and the Gauss curvatures. Furthermore, the trace-free part of $I_{1}$ vanishes to a finite order at every umbilic point.

According to Theorem 1, if an isometric embedding, $F_{1}$, admits a Bonnet mate, then the net formed by the curvature lines of $F_{1}$ has the same local character, that is, the same type of singularities as the net formed by the horizontal and vertical foliations of a holomorphic quadratic differential. Next we consider what can be said about an embedding whose foliations of curvature lines are precisely the horizontal (vertical) foliations of a holomorphic quadratic differential.

Theorem 2. Let $M$ be a closed oriented Riemann surface, and let $F_{1}:(M, g) \rightarrow$ $\mathbf{R}^{3}$ be an isometric embedding whose net of curvature lines is the net formed by the principal stretch foliations of a holomorphic quadratic differential. Then an isometric embedding $F_{2}:(M, g) \rightarrow \mathbf{R}^{3}$ is congruent to $F_{1}$ if and only if $F_{1} \circ F_{2}^{-1}$ is orientation preserving and $H_{1}=H_{2}$.

The proof of Theorem 2 also yields a theorem about immersions:

Theorem 3. Let $M$ be a closed oriented Riemann surface, and let $F_{1}:(M, g) \rightarrow$ $\mathbf{R}^{3}$ be an isometric immersion whose net of curvature lines is the net formed by the principal stretch foliations of a holomorphic quadratic differential. If $F_{1}$ admits a Bonnet mate, then its mean curvature is constant.

In particular, the shape of most globally isothermic embeddings of a closed surface is determined by the mean curvature. For example, if $M$ has genus one and $F_{1}$ is globally isothermic, or if $F_{1}(M)$ is a surface of revolution, then every other isometric embedding, $F_{2}$, such that $H_{1}=H_{2}$ must be congruent to $F_{1}$.

The unique continuation property implies the following theorem:

Theorem 4. Let $F_{1}, F_{2}:(M, g) \rightarrow \mathbf{R}^{3}$ be two isometric embeddings, then $F_{1} \sim F_{2}$ if and only if the following set of conditions is satisfied: (i) $F_{1} \circ F_{2}^{-1}$ is orientation preserving; (ii) $H_{1}=H_{2}$; (iii) there exists a conformal map $\varphi: S^{2} \rightarrow S^{2}$ such that $N_{1}=\varphi \circ N_{2}$.

The unique continuation also yields a generalization of the following classical result: If $H_{1}=H_{2}$ and $N_{1}=N_{2}$, then $F_{1}=$ translation $\circ F_{2}$; see [Gar]. 
Theorem 5. Let $F_{1}, F_{2}:(M, g) \rightarrow \mathbf{R}^{3}$ be two isometric embeddings, then $F_{1} \sim F_{2}$ if and only if the following set of conditions is satisfied: (i) $F_{1} \circ F_{2}^{-1}$ is orientation preserving; (ii) $H_{1}=H_{2}$; (iii) $\left(N_{1}-N_{2}\right)(M)$ lies in a half-space.

\section{DIRAC SPINORS AND CONFORMAL IMMERSIONS}

To obtain deeper rigidity results and to find and classify possible counterexamples to the rigidity conjecture one needs a new method for constructing surfaces. Such a theory is outlined here and then used to investigate the following modified rigidity conjecture:

Let $(M, g)$ be a closed and oriented Riemann surface. Given a function $H$ on $(M, g)$, and a regular homotopy class, $\mathcal{F}$, of immersions of $M$ into $\mathbf{R}^{\mathbf{3}}$, there exists at most one, up to rigid motions, isometric immersion $F \in \mathcal{F}$ with mean curvature function $H$.

Note that if the modified conjecture is false, then the rigidity conjecture from the previous section is also false.

Instead of a new method for constructing surfaces one could try to use the Weierstrass-Kenmotsu representation [Ken]. To apply this representation one must solve Kenmotsu's system of differential equations satisfied by the differential $d F$ of a conformal immersion $F$ with prescribed mean curvature function $H$. The main difficulty is that the system is nonhomogeneous, second order, and nonlinear if $H$ is nonconstant, and, in addition, the system is degenerate at points at which $H=0$.

The Weierstrass representation of minimal surfaces was reformulated in terms of spinors in [Sul]. These ideas were used in [KS], where the authors also indicated that there should be a theory for general, not necessarily minimal, surfaces. Indeed, such theories were developed in [Bob], and later in [Ko] (see also [KT]), and [Ri]. During the academic year 1995-1996 the GANG seminar at the University of Massachusetts set out to investigate the role of spinors in the geometry of immersed surfaces and to develop a general theory of the spinor representation of surfaces. The goal was to design an efficient and useful calculus, and to give a transparent explanation of the objects involved in the representation [Pi1, Pe, Ka1, KNPP].

From now on $M$ always denotes a Riemann surface, that is, an oriented surface with a chosen conformal structure. In particular, $M$ comes equipped with a maximal holomorphic atlas $\left\{\left(U_{\alpha}, z_{\alpha}\right)\right\}$. The conformal structure allows us to make sense of nonnegative two-forms, and their square roots, the nonnegative and the nonpositive half-densities. The square roots of a nonnegative two-form $w d x_{\alpha} \wedge d y_{\alpha}$ defined by $\pm \sqrt{w d x_{\alpha} \wedge d y_{\alpha}}= \pm \sqrt{w}\left|d z_{\alpha}\right|$ are sections in the fiber bundle $\mathcal{D}^{\frac{1}{2}}$ of halfdensities. Thus, the square root of a nonnegative two-form, in particular an area element, is a half-density, and vice versa, the square of a half-density is a two-form. Half-densities are necessary to introduce a conformally invariant notion of surface tension:

Definition 1. Let $F$ be a conformal immersion of $M$ into $\mathbf{R}^{3}$ inducing the area element $d A$ by pulling back the Euclidean metric by $F$, and let $H$ be the mean curvature function of the immersion $F$. The half-density $H \sqrt{d A}$ is called the mean curvature half-density of $F$.

A conformal immersion, $F$, of a Riemann surface into $\mathbf{R}^{\mathbf{3}}$ defines a spin structure on the Riemann surface. The spin structure characterizes uniquely the regular 
homotopy class of the immersion. See $[\mathrm{Pi}]$ and $[\mathrm{Sul}]$. The square root, $\sqrt{d F}$, of the differential of $F$ is a section in the associated spinor bundle, $\Sigma$.

For the rest of this paper we identify $\mathbf{R}^{\mathbf{3}}$ with the imaginary quaternions $\operatorname{im}(\mathbf{H})$. A spinor bundle on a Riemann surface is a quaternionic line bundle $\Sigma$ with transition functions $k_{\beta \alpha}: U_{\alpha} \cap U_{\beta} \rightarrow \mathbf{C}$ such that $k_{\beta \alpha}^{2}=\partial z_{\alpha} / \partial z_{\beta}$. A choice of a spinor bundle is equivalent to choosing a square root bundle of the bundle of conformal $\mathbf{R}^{3}$-valued one-forms on $M$. Indeed, the following theorem is known:

Theorem 6. A spinor bundle on a Riemann surface $M$ is a quaternionic line bundle $\Sigma$ on $M$ with a chosen endomorphism $J \in \operatorname{End}_{\mathbf{H}}(\Sigma)$ and a nontrivial quaternionic-hermitian, fiber-preserving pairing

$$
(\cdot, \cdot): \Sigma \times \Sigma \rightarrow T^{*} M \otimes \mathbf{H},
$$

so that $J^{2}=-1$, and for every two spinors $\psi, \phi \in \Sigma$ based at the same point $p$ we have

$$
(\phi, \psi)(J X)=(J \phi, \psi)(X)=(\phi, J \psi)(X)
$$

for every $X \in T_{p} M$. Here $J X$ denotes the action of the complex structure on the vector $X$.

Note that for every spinor $\psi \in \Sigma$ the form $(\psi, \psi)=\omega$ is imaginary quaternionic valued. Furthermore, $(\psi, \psi)$ is a conformal $\mathbf{R}^{\mathbf{3}}=\operatorname{im}(\mathbf{H})$ valued one-form on $M$. The spinor $\psi$ is interpreted as the square root of $\omega$. Every spinor $\psi$ defines a nonnegative half-density $|\psi|^{2}:=\|(\psi, \psi)\|$, where $\|\cdot\|$ is the Euclidean norm in $\mathbf{R}^{3}$. The half-density $|\psi|^{2}$ measures the relative dilation associated with the conformal form $(\psi, \psi)$. A choice of a spinor bundle is equivalent to a choice of a spin structure, that is, a holomorphic square root of the canonical bundle $T^{(1,0)} M^{*}$ of $M$. Indeed, given a spinor bundle $\Sigma$, define the complex line bundle of positive spinors by $\Sigma_{+} \stackrel{\text { def }}{=}\{\sigma \in \Sigma \mid J \sigma=\sigma i\}$. For every positive spinor $\sigma \in \Sigma_{+}$we define the one-form $\sigma^{2} \stackrel{\text { def }}{=}-k(\sigma, \sigma)$. From (1) it follows that $\sigma^{2}(J X)=i \sigma^{2}(X)=\sigma^{2}(X) i$ for every vector $X$. Thus, for every positive spinor $\sigma$, the one-form $\sigma^{2}$ is complex valued and belongs to the canonical bundle $T^{(1,0)} M^{*}$ of $M$. The map sending $\sigma \in \Sigma_{+}$to $\sigma^{2}$ is a quadratic map from $\Sigma_{+}$onto the canonical bundle of $M$. Thus every $\psi \in \Sigma_{+}$ defines a conformal one-form $\psi^{2} \in T^{(1,0)} M^{*}$ and a nonnegative half-density $|\psi|^{2}$.

There is a canonical bi-additive fiberwise pairing $\Sigma \times\left(T^{*} M \otimes_{R} \mathbf{H}\right) \rightarrow \mathcal{D}^{\frac{1}{2}} \otimes_{\mathbf{R}} \Sigma$ which defines conformal Clifford product between spinors and $\mathbf{H}$-valued one-forms. The conformal Dirac operator is the unique local linear operator

$$
D: \Gamma(\Sigma) \rightarrow \Gamma\left(\mathcal{D}^{\frac{1}{2}} \otimes \Sigma\right)
$$

satisfying the Leibniz rule and such that $D \sigma=0$ if $\sigma$ is a local section of $\Sigma_{+}$whose square is a closed one-form, i.e., if $d \sigma^{2}=0$. (Compare with [Hit] and [At].)

Definition 2. A section, $\psi \in \Gamma(\Sigma)$, is called a Dirac spinor if $D \psi=U \otimes \psi$ for some $U \in \Gamma\left(\mathcal{D}^{\frac{1}{2}}\right)$; we say that $\psi$ generates the half-density $U$.

Dirac spinors represent the square roots of the differentials of conformal immersions:

Theorem 7. For every nonvanishing $\psi \in \Gamma(\Sigma)$ the one-form $(\psi, \psi)$ is closed if and only if $\psi$ is a Dirac spinor. If $M$ is simply connected and $\psi$ is a nonvanishing 
Dirac spinor, then

$$
F=\int(\psi, \psi)
$$

is a conformal immersion with mean curvature half-density equal to the half-density generated by $\psi$. Vice versa, given an oriented surface $M$, every immersion $F$ of $M$ into $\mathbf{R}^{\mathbf{3}}$ defines a unique, up to isomorphism, spinor bundle $\Sigma$ on the surface $M$ equipped with the pull-back conformal structure and precisely two Dirac spinors, $\psi$ and $-\psi$, such that $( \pm \psi, \pm \psi)=d F$. The half-density generated by $\pm \psi$ equals the mean curvature half-density of $F$.

Note that in fact $\Sigma=M \times \mathbf{H}$ and after identifying $\mathbf{R}^{\mathbf{3}}$ with $\operatorname{im}(\mathbf{H})$ we have $(\psi, \phi)=\bar{\psi} d F \phi$, where $\psi$ and $\phi$ are spinors based at the same point on $M$. I will denote by $[\Sigma]$ the regular homotopy class of an immersion inducing the spinor bundle $\Sigma$.

The standard elliptic theory and the representation (2) imply the following local existence result: Every half-density can be realized locally as the mean curvature half-density of a conformal immersion. Global existence and the Dirichlet problem are discussed in [Ka3] and [Ka4].

The outlined theory provides new insight into Bonnet's problem. Given a metric $g$ on $M$ and a function $H \neq$ const, there exists at most one, up to rigid motions, Bonnet pair $F_{ \pm}$of isometric immersions of $(M, g)$ into $\mathbf{R}^{\mathbf{3}}$ with mean curvatures $H_{ \pm}=H([\mathrm{LT}])$. I am interested in the space of geometrically distinct pairs of Bonnet mates on a given Riemann surface $M$ and within the same regular homotopy class. The space of geometrically distinct pairs of Bonnet mates is the space of Bonnet pairs modulo the natural gauge group $\mathcal{G}=\mathcal{E}(3) \times \mathcal{E}(3) \times \mathbf{R}^{+}$acting on it. Here $\mathcal{E}(3)$ is the Euclidean group of rigid motions. Indeed, if we rotate one of the Bonnet mates in the pair $F_{ \pm}$we obtain another pair of Bonnet mates which is geometrically identical with the original pair $F_{ \pm}$. Furthermore, for every positive number $r>0$ the Bonnet pair $r F_{ \pm}$is simply the original pair $F_{ \pm}$observed at a different scale; thus, the pairs $F_{ \pm}$and $r F_{ \pm}$are not geometrically distinct. The action of the group $\mathcal{G}$ preserves the conformal class, the mean curvature half-density, and the regular homotopy class. Let $\mathcal{B}(M, \Sigma, U)$ be the moduli space of geometrically distinct pairs of Bonnet mates defined on the Riemann surface $M$ which belong to the regular homotopy class associated with the spinor bundle $\Sigma$ and induce a given potential $U$.

Theorem 8 ([Ka3]). For every half-density $U$ and regular homotopy class $[\Sigma]$ on the closed Riemann surface $M$, the moduli space $\mathcal{B}(M, \Sigma, U)$ of geometrically distinct Bonnet mates is either empty or it is a disjoint union of isolated components. Each nonempty component is either a point, a line, or a four-dimensional ball.

Note that $\operatorname{dim}_{\mathbf{H}} \operatorname{ker}(D-U)$ is finite if $M$ is closed. If $\operatorname{dim}_{\mathbf{H}} \operatorname{ker}(D-U)=1$, then $\mathcal{B}(M, \Sigma, U)$ is empty. Furthermore:

Theorem 9 ([Ka3]). Suppose that $U$ is a half-density on the Riemann surface $M$; if the spinor bundle $\Sigma$ is such that $\operatorname{dim}_{\mathbf{H}} \operatorname{ker}(D-U)=2$, then precisely one of the following alternatives holds:

(a) $\mathcal{B}(M, \Sigma, U)=\emptyset$.

(b) $\mathcal{B}(M, \Sigma, U)$ is a point.

(c) $\mathcal{B}(M, \Sigma, U)$ is homeomorphic to a line segment. 
(d) $\mathcal{B}(M, \Sigma, U)$ is homeomorphic to a four-dimensional ball.

Furthermore, every conformal immersion that belongs to the regular homotopy class $[\Sigma]$ and induces the mean curvature half-density $U$ admits at most one, up to rigid motions, Bonnet mate.

Theorem 9 provides an extension of the results from [LT] including constant mean curvature immersions.

Corollary 2.1. Let $(M, g)$ be an oriented, closed Riemann surface, and let $H$ be a function defined on $M$, possibly constant. Every regular homotopy class $[\Sigma]$ such that $\operatorname{dim}_{\mathbf{H}} \operatorname{ker}(D-U)=2$ contains at most two geometrically distinct isometric immersions of $(M, g)$ into $\mathbf{R}^{3}$ with mean curvature function $H$.

Remark. One can draw a parallel between Bonnet's rigidity conjecture and Pauli's exclusion principle by thinking of the Dirac spinor $\psi$ of an immersion $f$ as a wave function. Indeed, the Dirac spinor satisfies the equation $D \psi=U \psi$ and defines the half-density $|\psi|^{2}=\sqrt{d A}$, where $d A$ is the area element induced on the surface by the immersion $f$. Two noncongruent immersions $f_{ \pm}$are Bonnet mates if and only if they are in the same conformal class and induce the same area element and the same mean curvature half-density $U_{+}=U_{-}$. Thus if we consider only Bonnet mates within the same regular homotopy class, then two noncongruent immersions $f_{ \pm}$are Bonnet mates if and only if their respective Dirac spinors $\psi_{ \pm}$generate the same potential $U_{+}=U_{-}$and the same half-density $\left|\psi_{+}\right|^{2}=\left|\psi_{-}\right|^{2}$. Furthermore, it is natural to think that the Dirac spinors $\psi$ and $\psi \alpha$, where $\alpha$ is a unit quaternion, represent the wave functions of the same particle, that is, the same quantum state, with respect to two different Euclidean reference frames in the ambient space $\mathbf{R}^{\mathbf{3}}$. Hence the modified rigidity conjecture is equivalent to saying that the quantum state is uniquely determined by the potential (energy) and the position density.

\section{REFERENCES}

[At] M. Atiyah, Riemann surfaces and spin structures, Ann. Sci. Ec. Norm. Sup. 4 (1971), 47-62. MR 44:3350

[Bob] A. I. Bobenko, Surfaces in terms of 2 by 2 matrices, Harmonic Maps and Integrable Systems (A. Froddy and J. Wood, eds.), Aspects of Mathematics, Vieweg, Braunschweig, 1994. MR 95m:58047

[Bon] O. Bonnet, Mémoire sur la théorie des surfaces applicables, J. Éc. Polyt. 42 (1867).

[Car] É. Cartan, Sur les couples de surfaces applicables avec conservation des courbures principales, Bull. Sc. Math. 66 (1942), 55-72, 74-85. MR 5:216e

[Che] S. S. Chern, Deformations of surfaces preserving principal curvatures, Differential Geometry and Complex Analysis: a Volume Dedicated to the Memory of H. E. Rauch (I. Chavel and H. M. Farkas, eds.), Springer-Verlag, 1985, pp. 155-163. MR 86h:53005

[Gar] R. Gardner, An integral formula for immersions in Euclidean space, J. Diff. Geom. 3 (1969), 245-252. MR 40:7992

[Hit] N. Hitchin, Harmonic spinors, Adv. in Math. 14 (1974), 1-55. MR 50:11332

[Hopf] H. Hopf, Differential geometry in the large, Lecture Notes in Mathematics, vol. 1000, Springer-Verlag, Berlin and New York, 1983. MR 85b:53001

[Ka] G. Kamberov, Recovering the shape of a surface from the mean curvature, to appear in Proc. of the Intl. Conf. on Differential Equations and Dynamical Systems, 1996, publ. by Marcel Dekker, (1998).

[Ka1] G. Kamberov, Lectures on quaternions, spinors, and surfaces, Notes from the lectures at the University of São Paulo, Brazil, May-June, 1997.

[Ka2] G. Kamberov, Quadratic differentials, quaternionic forms, and surfaces, preprint.

[Ka3] G. Kamberov, Immersions of closed surfaces with prescribed mean curvature halfdensity, in preparation. 
[Ka4] G. Kamberov, The Dirichlet problem for Dirac spinors, preprint.

[Ken] K. Kenmotsu, Weierstrass formula for surfaces of prescribed mean curvature, Math. Ann. 245 (1979), 89-99. MR 81c:53005b

[KNPP] G. Kamberov, P. Norman, F. Pedit, U. Pinkall, Surfaces, quaternions, and spinors, preprint.

[KPP] G. Kamberov, F. Pedit, and U. Pinkall, Bonnet pairs and isothermic surfaces, GANG Preprint IV.21, 1996, to appear in Duke Math. J. 92 (1998).

[Ko] B. G. Konopelchenko, Induced surfaces and their integrable dynamics, Stud. Appl. Math. 96 (1996), 9-51. MR 96i:53011

[KT] B. G. Konopelchenko and I. A. Taimanov, Generalized Weierstrass formulae, soliton equations and the Willmore surfaces, I. Tori of revolution and the $m K D V$, preprint no. 187, Ruhr-Universität-Bochum, Fakultät für Mathematik, 1995.

[KS] R. Kusner and N. Schmidt, The spinor representation of minimal surfaces, GANG Preprint III.27, 1993. Revised version, GANG Preprint 4.18.

[LT] B. Lawson and R. Tribuzy, On the mean curvature function for compact surfaces, J. Diff. Geom. 16 (1981), 179-183. MR 83e:53060

[Pe] F. Pedit, Lectures at the Summer School in Differential Geometry, Durham, UK, Summer 1996.

[Pi] U. Pinkall, Regular homotopy classes of immersed surfaces, Topology 24 (1985), 421435. MR 87e:57028

[Pi1] U. Pinkall, Lectures at TU Berlin, Spring 1996.

[Ri] J. Richter, Surfaces in terms of spinors, Master Thesis, TU Berlin, 1995.

[Ros] A. Ros, Compact hypersurfaces with constant scalar curvature and a congruence theorem, J. Diff. Geom. 27 (1988), 215-223. MR 89b:53096

[Sul] D. Sullivan, The spinor representation for minimal surfaces in space, Unpublished notes, 1989.

Department of Mathematics, Washington University, St. Louis, MO

E-mail address: kamberov@math.wustl.edu 